Radial and Nonradial Pulsations as Probes of Stellar Physics

ASP Conference Series, Vol. 259, 2002

C. Aerts, T.R. Bedding, \& J. Christensen-Dalsgaard, eds.

\title{
$\delta$ Sct-Type Pulsators in Eclipsing Binary Systems: the Case of RZ Cas
}

E. Rodríguez, V. Costa, M.J. López-González

Instituto de Astrofísica de Andalucia, CSIC, P.O. Box 3004, E-18080 Granada, Spain

\section{J.M. García}

Departamento de Fúsica, E.U.I.T. Industriales, UPM, Ronda de Valencia 3, E-28012 Madrid, Spain

S.L. Kim, J.W. Lee, J.H. Yoon

Korea Astronomy Observatory, Taejon, 305-348, Korea

E. Hintz

Department of Physics and Astronomy, Brigham Young University, Provo, UT 84602, USA

D.E. Mkrtichian, A.Y. Gamarova

Astronomical Observatory, Odessa State University, Shevchenko Park, Odessa, 270014, Ukraine

A.V. Kusakin

Sternberg State Astronomical Institute, Universitetsky prospect, 13, Moscow, 119899, Russia

\begin{abstract}
RZ Cas is an Algol-type eclipsing binary system where the primary component was recently discovered as a $\delta$ Sct pulsator. A threecontinent multisite photometric campaign was carried out during 1999. Preliminary results are reported here indicating a semi-detached system where the secondary fills its Roche lobe. The light curves also suggest a hot spot on the surface of the primary component as a consequence of the impact of the mass stream from the secondary. The pulsational behaviour can be well described with only one frequency.
\end{abstract}

\section{Discussion}

RZ Cas is one of the very few examples known to date of an eclipsing binary system with one component as a $\delta$ Sct-type pulsating star (Rodríguez \& Breger, 2001). In this case, we are dealing with an Algol-type system (A3V+K0IV) where the primary component is the pulsating one. The orbital period is $\mathrm{P}_{b}=1 .^{d} 1953$, with eclipse depths in the primary and secondary minima of about 
$\Delta V \sim 1 .^{m} 50$ and $0 \cdot^{m} 07$, respectively. The pulsational amplitude is very small $\left(\sim 0{ }^{m} 01\right)$ with a very short main period (22 minutes) (Ohshima et al., 1998), but the pulsational behaviour is directly shown in the observed light curves, even during the secondary minimum and at the beginning or end of the primary minimum. However, the pulsational history of this system is very short and only the work by Ohshima et al. (1998) is available to date.

In order to make a detailed study of the pulsational behaviour of the primary component, a multisite photometric campaign was carried out during the autumn of 1999 including observatories from Asia (Korea and Kazakhstan), Europe (Spain) and America (USA). The observations from Asia and America were collected using CCD photometry and mainly single $V$ Johnson filter, whereas simultaneous observations in the four uvby filters of the Strömgren photometric system were collected at Sierra Nevada, Spain. When the uvby light curves and corresponding colour index variations are phased with the orbital period (2457 simultaneous uvby points were collected and nearly all orbital phases have been covered at least three times), some very interesting features are shown: 1) The observed curves are not completely symmetric; this is especially shown in the $v$ filter where the high level at second quadrature is higher than at the first one; this is also shown in the $(b-y)$ and $c_{1}$ curves. This suggests a hot spot on the surface of the primary component as a result of the (impact of the) mass stream from the secondary. 2) On the other hand, the $m_{1}$ index decreases during the primary minimum. This should mean chromospheric activity in the secondary component.

In order to investigate the pulsational properties of the primary component, the orbital light variations due to binarity have to be removed from the observed light curve. The shape of the underlying binary curve corresponds to that of an Algol-type eclipsing binary system and the binarity was solved using the WilsonDevinney code as the most appropriate for modelling the curves of such system. After some preliminary tests, we assumed this system as a semi-detached one where the secondary fills its Roche lobe, the orbit is circular and there is no third light. The model also includes a hot spot on the surface of the primary located on the equator of the primary. After removing the computed binary light curve, the pulsation of the primary component was analysed in order to investigate the frequency content. The frequency analysis was carried out using the method described in Rodríguez et al. (1998), where single-frequency and multiple-frequency techniques are combined using both Fourier and multiple least-squares algorithms. However, the periodograms only show one significant peak at $\nu=64.1942 \mathrm{c} \mathrm{d}^{-1}$. After prewhitening for this frequency, the resulting periodograms did not show any trace of another peak, suggesting a monoperiodic nature for this star.

\section{References}

Ohshima, O., Narusawa, S.Y., Azakawa, H., et al. 1998, IBVS, 4581

Rodríguez, E. \& Breger, M. 2001, A\&A, 366, 178

Rodríguez, E., Rolland, A., López-González, M.J., \& Costa, V. 1998, A\&A, 338, 905 